\title{
MATRIX ELEMENTS FOR THE ONE-DIMENSIONAL HARMONIC OSCILLATOR
}

\author{
José L. López-Bonilla \& G. Ovando
}

\begin{abstract}
The properties of the Hermite polynomials are used to obtain the matrix elements $\left\langle m\left|e^{-\gamma x}\right| n\right\rangle$ for the harmonic oscillator in one dimension in an easy way.
\end{abstract}

\section{Introduction}

One month before E. Schrödinger published his famous equation, the Hungarian physicist C. Lanczos [1-6] wrote an integral equation as the first non-matricial version of quantum mechanics. If we transform the integral equation into a differential one, there results the Schrödinger equation for stationary states:

$$
\left[-\frac{\hbar^{2}}{2 M} \frac{d^{2}}{d x^{2}}+V(x)\right] \Psi_{n}(x)=E_{n} \Psi_{n}(x) .
$$

The harmonic oscillator (HO), with potential $V(x)=\frac{M}{2} \omega^{2} x^{2}$, was one of the first cases solved on in an exact way [7]. The corresponding solution appears in any book on quantum mechanics. It is given by (we will employ natural units such that $\hbar=M=\omega=1$ ):

$$
\begin{gathered}
\Psi_{n}(x)=\frac{1}{\sqrt{2^{n} n !} \sqrt{\pi}} e^{-\frac{x^{2}}{2}} H_{n}(x), \quad-\infty<x<\infty, \\
E_{n}=n+\frac{1}{2}, \quad n=0,1,2, \ldots
\end{gathered}
$$

where the $H_{n}(x)$ stands for the Hermite polynomials [8]:

$$
H_{n}(x)=(-1)^{n} e^{x^{2}} \frac{d^{n}}{d x^{n}} e^{-x^{2}}
$$


satisfying the orthonormality property

$$
\int_{-\infty}^{+\infty} e^{-x^{2}} H_{p}(x) H_{q}(x) d x=2^{p} p ! \sqrt{\pi} \delta_{p, q}
$$

The goal of this paper is to use an analytical method to calculate the following matrix elements for the $\mathrm{HO}$ :

$$
\left\langle m\left|e^{-\gamma x}\right| n\right\rangle \equiv \int_{-\infty}^{+\infty} \Psi_{m}(x) e^{-\gamma x} \Psi_{n}(x) d x, \quad \gamma \geq 0
$$

in an alternative procedure compared to other methods presented in the literature [9-13]. In [10] it is shown how with equation (3) it is easy to obtain $\left\langle m\left|x^{k}\right| n\right\rangle, k=0,1,2, \ldots$, which leads to closed formulas for $\langle m|f(x)| n\rangle$ using the Taylor series of $f(x)$.

In the next section we will determine (3) explicitly; our procedure is very simple and follows known mathematical relations involving the Hermite polynomials. We hope that this technique may be useful for those people interested in quantum mechanics.

\section{Matrix elements for the $\mathrm{HO}$}

Because expression (3) is symmetrical in the indices $m$ and $n$, we can take $m \geq n$ without loss of generality. The $H_{n}$ are a basis because any function is a series expansion of them, in particular from [8] we have

$$
e^{-\gamma x}=e^{\frac{\gamma^{2}}{4}} \sum_{r=0}^{\infty} \frac{(-\gamma)^{r}}{2^{r} r !} H_{r}(x)
$$

which in conjunction with (2.a) and (3) implies

$$
\left\langle m\left|e^{-\gamma x}\right| n\right\rangle=\left(2^{n+m} \pi n ! m !\right)^{-1 / 2} e^{\frac{\gamma^{2}}{4}} \sum_{r=0}^{\infty} \frac{(-\gamma)^{r}}{2^{r} r !} I(r, n, m)
$$

where $\quad I(r, n, m)=\int_{-\infty}^{\infty} e^{-x^{2}} H_{r}(x) H_{n}(x) H_{m}(x) d x$. 
But the product of two Hermite polynomials is another polynomial admitting an expansion in terms of $H_{n}$, see [8]:

$$
H_{n}(x) H_{m}(x)=2^{n} n ! m ! \sum_{k=0}^{n} \frac{H_{2 k+m-n}(x)}{2^{k} k !(k+m-n) !(n-k) !}
$$

whose insertion into (4.b) leads to

$$
\left\langle m\left|e^{-\gamma x}\right| n\right\rangle=\left(2^{n-m} \frac{n ! m !}{\pi}\right)^{1 / 2} e^{\frac{\gamma^{2}}{4}} \sum_{r=0}^{\infty} \frac{(-\gamma)^{r}}{2^{r} r !} J(r, n, m),
$$

where

$$
\begin{aligned}
& J(r, n, m)= \\
& \sum_{k=0}^{n} \frac{1}{2^{k} k !(k+m-n) !(n-k) !} \int_{-\infty}^{\infty} e^{-x^{2}} H_{r}(x) H_{2 k+m-n}(x) d x .
\end{aligned}
$$

This allows us to use (2.c) to obtain

$$
\left\langle m\left|e^{-\gamma x}\right| n\right\rangle=\left(2^{n-m} \frac{n !}{m !}\right)^{1 / 2} e^{\frac{\gamma^{2}}{4}}(-\gamma)^{m-n} f(n, m)
$$

where $\quad f(n, m)=\sum_{k=0}^{n} \frac{m ! \gamma^{2 k}}{2^{k} k !(k+m-n) !(n-k) !}$.

On the other hand, the associated Laguerre polynomials $L_{n}^{q}(x)$, see [14], are defined by

$$
L_{n}^{q}(x)=\sum_{k=0}^{n} \frac{(-1)^{k}(n+q) !}{k !(k+q) !(n-k) !} x^{k}
$$

Evaluating this polynomial when $q=m-n$ and $x=-\frac{\gamma^{2}}{2}$ gives

$$
L_{n}^{m-n}\left(-\frac{\gamma^{2}}{2}\right)=\sum_{k=0}^{n} \frac{m ! \gamma^{2 k}}{2^{k} k !(k+m-n) !(n-k) !} x^{k}
$$

and leads to an expression of (5.b) as 


$$
\left\langle m\left|e^{-\gamma x}\right| n\right\rangle=\left(2^{n-m} \frac{n !}{m !}\right)^{1 / 2}(-\gamma)^{m-n} e^{\frac{\gamma^{2}}{4}} L_{n}^{m-n}\left(-\frac{\gamma^{2}}{2}\right),
$$

in complete accordance with the expressions of other authors $[10,12,15]$.

It is known from [10] that with equation (7) we are able to determine the matrix elements

$$
\left\langle m\left|x^{q}\right| n\right\rangle \equiv \int_{-\infty}^{+\infty} \Psi_{m}(x) x^{q} \Psi_{n}(x) d x, \quad q=0,1,2, \ldots
$$

however, if we desire to calculate (8.a) directly (for $q$ even or odd), we would employ the following expansions (see [8])

$$
x^{2 p}=\frac{(2 p) !}{2^{2 p}} \sum_{k=0}^{p} \frac{H_{2 k}(x)}{(2 k) !(p-k) !}
$$

and $x^{2 p+1}=\frac{(2 p+1) !}{2^{2 p+1}} \sum_{k=0}^{p} \frac{H_{2 k+1}(x)}{(2 k+1) !(p-k) !}, \quad p=0,1,2, \ldots$ (8.c) and then repeat the process shown in this paper. This results in the formulas for (8.a) reported in the literature, see [9-11,13].

\section{References}

[1] C. Lanczos, On a field theoretical representation of the new quantum mechanics, Z. Phys. 35 (1926), 812.

[2] J. Mehra, Aspects of Quantum Theory, ed. A. Salam, E. Wigner. Cambridge University Press, 1972.

[3] J. Mehra, The Physicist's Conception of Nature. Academic Press, 1974.

[4] B. K. P. Scaife, Studies in Numerical Analysis. Academic Press, 1974.

[5] B. Gellai, Cornelius Lanczos, Comp. Maths. Appls. 1 (1975), 260.

[6] I. Rhodes, Reminicences of a cherished friend, Comp. Maths. Appls. 1(1975), 267. 
[7] C. Eckart, The solution of the problem of the simple oscillator by a combination of the Schrödinger and Lanczos theories, Proc. Nat. Acad. Sc. 12 (1926), 473.

[8] N. N. Lebedev, Special Functions and their Applications. Dover: New York, 1965.

[9] J. Morales, A. Palma and L. Sandoval, Hypervirial theorem and ladder operators. Recurrence relations for harmonic oscillator integrals, Int. J. Quantum Chem. 29 (1986), 211.

[10] J. López-Bonilla, J. Morales and A. Palma, Hypervirial theorem and parameter differentiation: closed formulation for harmonic oscillator integrals, J. Math. Phys. 28(1987), 1032.

[11] A. Palma, L. Sandoval and J. Morales, Closed formulas for one and two center harmonic oscillator integrals, Int. J. Quantum Chem. S21(1987), 729.

[12] V. Gaftoi, J. López-Bonilla, J. Morales and D. Navarrete, Integral representation of the Laguerre polynomials, Rev.Mex.Fis 36 (1990), 310

[13] O. L. de Lange and R. E. Raab, Operators Methods in Quantum Mechanics. Clarendon Press: Oxford, 1991.

[14] M. Abramowitz and I. A. Stegun, Handbook of Mathematical Functions. John Wiley and Sons: New York, 1972.

[15] J. López-Bonilla, D. Navarrete, H. N. Núñez-Yépez and A. L. SalasBrito, Oscillators in one and two dimensions and ladder operators for the Morse and the Coulomb problems, Int. J. Quantum Chem. 62 (1997), 177.

José L. López-Bonilla

The International Institute of Integral Human Sciences

1974 de Maisonneuve West

Montréal, Québec

Canada H3H $1 \mathrm{~K} 5$

G. Ovando

Departamento de Ciencias Básicas

Universidad Autónoma Metropolitana-Azcapotzalco

Apdo. Postal 16-306

02200 México D.F.

e-mail: gaoz@hp9000a1.uam.mx 